\title{
First-degree family history of diabetes and its relationship with serum osteocalcin levels independent of liver fat content in a non-diabetic Chinese cohort
}

\author{
Yiting $\mathrm{Xu}^{\dagger}$, Yun Shen ${ }^{\dagger}$, Xiaojing $\mathrm{Ma}^{*}$, Chengchen Gu, Yufei Wang and Yuqian Bao*
}

\begin{abstract}
Background: First-degree relatives of patients with diabetes (FDR) tend to have impaired insulin activity, which lead to the alternation of circulating cytokine levels. Liver is a main target tissue of insulin action; therefore, liver fat content (LFC) has a close relationship with insulin resistance. This study aimed to find the alteration in serum osteocalcin levels in FDR and the relationship of serum osteocalcin levels with FDR and non-alcoholic fatty liver disease (NAFLD).

Methods: In total, 1206 subjects including 413 men and 793 women from the communities, aged 59.7 (range, 54.8-64.3) years, were enrolled. An electrochemiluminescence immunoassay was performed to measure the levels of serum osteocalcin. LFC was measured using quantitative ultrasonography.

Results: A significant decrease was found in serum osteocalcin levels in subjects with NAFLD $(P<0.001)$ as well as in FDR $(19.8 \pm 5.7 \mathrm{ng} / \mathrm{mL}$ versus $20.7 \pm 6.8 \mathrm{ng} / \mathrm{mL}, P=0.028)$. Furthermore, among the subjects with NAFLD, those with FDR had lower levels of osteocalcin than those without FDR $(P=0.011)$. The presence of FDR remained a predictor for decreased serum osteocalcin levels after adjusting for body mass index, blood glucose, blood lipids, and LFC (standardized $\beta=-0.057, P=0.028$ ).
\end{abstract}

Conclusions: FDR had lower serum osteocalcin levels than non-FDR. The inverse association between FDR and serum osteocalcin levels was independent of metabolic factors.

Keywords: First-degree relatives of patients with diabetes, Osteocalcin, Liver fat content, Insulin resistance

\section{Background}

Genetic defects play an important role in the progression of diabetes in susceptible individuals. A few specific genetic abnormalities predisposed to impaired insulin action in vivo were confirmed in a preliminary study [1]. Accumulating evidence suggests that the genetic background of first-degree relatives of patients with diabetes (FDR) has an effect on insulin resistance, leading to a high risk of metabolic complications such as overweight/

\footnotetext{
* Correspondence: maxiaojing@sjtu.edu.cn; yqbao@sjtu.edu.cn

${ }^{\dagger}$ Yiting Xu and Yun Shen contributed equally to this work.

Department of Endocrinology and Metabolism, Shanghai Jiao Tong

University Affiliated Sixth People's Hospital; Shanghai Clinical Center for Diabetes; Shanghai Key Clinical Center for Metabolic Disease; Shanghai Diabetes Institute; Shanghai Key Laboratory of Diabetes Mellitus, Shanghai 200233, China
}

obesity, hyperglycaemia, and dyslipidaemia [2, 3]. Moreover, genetic defects in FDR can regulate the transcription of cytokine genes, resulting in abnormalities of the secretion and metabolism of cytokines [4]. Our previous studies have found that the levels of adipokines in FDR are significantly increased, possibly because of impaired insulin function [5].

Bone has recently emerged as an endocrine organ that secretes a relatively abundant non-collagen protein, osteocalcin. This small peptide is primarily produced by osteoblasts and partly released into blood, where its concentration serves as a hormone implicated in the regulation of glucose and lipid metabolism, improving insulin sensitivity and stimulating insulin secretion as well [6-10]. Liver is the main site of insulin resistance. 
Our previous study reported that subjects with nonalcoholic fatty liver disease (NAFLD) diagnosed by ultrasound had significantly decreased serum osteocalcin levels. In addition, serum osteocalcin levels were closely related to fatty liver index, which was estimated based on laboratory tests $[11,12]$. However, quantitative measurement by ultrasonography has higher sensitivity than traditional methods in detecting mild hepatic steatosis. Not only can it accurately quantify liver fat content (LFC) but it can also reduce the interobserver and intraobserver variability, and therefore, its use in clinical practice might help to identify subjects with an increased risk for metabolic diseases [13, 14].

As there is lack of evidence focused on the relationship between FDR and serum osteocalcin levels, the present study was designed to investigate alterations in serum osteocalcin levels in FDR. Ultrasound quantification was applied to detect LFC to evaluate changes in serum osteocalcin levels in FDR under the influence of liver steatosis.

\section{Methods \\ Subjects}

This study included non-diabetic individuals from communities in Shanghai, more details could found in our previous study [15]. All subjects underwent questionnaire, physical and biochemical measurement in the study. Those with positive for either hepatitis B surface antigen or anti-hepatitis $C$ virus antibody, excessive alcohol consumption in the past 12 months (men: $\geq 210 \mathrm{~g}$ per week; women: $\geq 140 \mathrm{~g}$ per week), therapies that would influence the progression of NAFLD and/or osteocalcin levels (e.g., recent fractures, steroids, thyroxine) were excluded from the study; besides, the exclusion also included diabetes or receiving anti-hyperglycaemic therapy, history of cardiovascular disease, malignancy, thyroid dysfunction, and renal dysfunction. Ultimately, 1206 subjects were recruited with a finished questionnaire that gathered information on past and current medical history as well as current use of medications. A family history of diabetes was collected from responses to the systematic self-reported questionnaire. FDR was defined as individuals who have one or more first-degree relatives (parents, siblings, or children) diagnosed as having diabetes [16].

\section{Anthropometric and biochemical measurements}

Anthropometric assessments such as body weight, height, blood pressure and waist circumference (W) were measured based on standard methods as previously described [17].

After an overnight fast of at least $10 \mathrm{~h}$, all subjects received a biochemical examination. A fasting blood sample was collected from each subject, and they also received a 75-g oral glucose tolerance test to measure 2$\mathrm{h}$ plasma glucose (2hPG). The laboratory test indicators were measured according to previous study, and further included liver enzymes [serum alanine aminotransferase (ALT), aspartate aminotransferase (AST), gammaglutamyl transpeptidase (GGT)], and serum osteocalcin levels. Laboratory testing methods have been described in previous studies [17]. The homeostasis model assessment-insulin resistance (HOMA-IR) index = fasting serum insulin $($ FINS $)(\mathrm{mU} / \mathrm{L}) \times$ fasting blood glucose (FPG) $(\mathrm{mmol} / \mathrm{L}) / 22.5$. An electrochemiluminescence immunoassay was used to determine serum osteocalcin levels (Roche Diagnostics, Mannheim, Germany) on a Roche Elecsys 2010 immunoassay analyser, with the intra- and interassay coefficients of variation as 1.2 to $4.0 \%$ and 1.7 to $6.5 \%$, respectively [6].

\section{Quantitative ultrasonography}

An abdominal ultrasonographic examination was performed in all subjects by a trained doctor specialized in ultrasonography, who was unaware of the study design and clinical details of the participants. The details of device were reported in previous study [12].

All the instrument settings, including "gain", "depth", etc. were calibrated using a tissue-mimicking phantom (model 057; Computerized Imaging Reference Systems, Norfolk, VA). The regions of interest in the captured images under the ultrasound machine were analysed using the image software certified by the National Institute of Health (Image 1.41o, National Institutes of Health, Bethesda, MD, USA) as detailed elsewhere [13]. The LFC was then obtained based on the following equation: $62.592 \times$ liver-kidney echo ratio $+168.076 \times$ liver attenuation coefficient -27.863 [13]. The subjects were defined as NAFLD if their LFCs by quantitative ultrasonography were $\geq 9.15 \%$ [13].

\section{Definitions}

Diabetes was defined as $\mathrm{FPG} \geq 7.0 \mathrm{mmol} / \mathrm{L}$ and/or $2 \mathrm{hPG} \geq 11.1 \mathrm{mmol} / \mathrm{L}$; impaired glucose regulation was diagnosed when $6.1 \mathrm{mmol} / \mathrm{L} \leq \mathrm{FPG}<7.0 \mathrm{mmol} / \mathrm{L}$ and/or $7.8 \mathrm{mmol} / \mathrm{L} \leq 2 \mathrm{hPG}<11.1 \mathrm{mmol} / \mathrm{L}$ [18]. Menopausal status was defined as continuous 12-month amenorrhea without other medical causes.

\section{Statistical analyses}

Statistical analyses were performed by SPSS version 20.0 (SPSS Inc., Chicago, IL, USA). Data with normally distributed are expressed in terms of the mean \pm standard deviation, whereas skewed data are expressed as the median with interquartile range. Numbers with percentages were used to express categorical variables. The differences between the two independent groups for normally distributed data, skewed data and categorical variables 
were assessed by using $t$-tests, Wilcoxon rank-sum test and chi-square test, respectively. Pearson correlation analysis as well as liner regression analysis was conducted to determine the associations between serum osteocalcin levels with LFC. One-way analysis of variance was used for trend analyses of serum osteocalcin in the FDR and NAFLD groups. Multivariable liner regression analysis was performed to examine the relationship of serum osteocalcin levels and FDR. All reported $P$ values were two-tailed, and $P<0.05$ was considered statistically significant.

\section{Results}

\section{Characteristics of the study participants}

A total of 413 men and 793 women, aged 59.7 (54.864.3) years, were enrolled in this study. Overall, serum osteocalcin levels were $20.5 \pm 6.6 \mathrm{ng} / \mathrm{mL}$, and the median LFC was 8.2 (6.6-22.2\%). The proportions of subjects with a family history of diabetes or NAFLD were $20.6 \%$ (249 patients) and 39.7\% (479 patients), respectively. As shown in Table 1, the prevalence of impaired glucose regulation was higher in FDR than non-FDR (48.6\% versus $41.0 \%, P=0.030$ ); however, there was no difference in the prevalence of NAFLD between FDR and non-FDR $(P=0.873)$. Serum osteocalcin levels significantly decreased in subjects with NAFLD than in those without $(19.7 \pm 6.2 \mathrm{ng} / \mathrm{mL}$ versus $21.1 \pm 6.8 \mathrm{ng} / \mathrm{mL}, P<0.001)$.

\section{Association between LFC and serum osteocalcin levels}

Correlation analysis revealed a significant negative relationship between LFC and serum osteocalcin levels $(r=$ $-0.114, P<0.001)$. Linear regression analysis revealed an independent and negative association between LFC and serum osteocalcin levels after adjusting for age, gender, and menopausal status (in women) (standardized $\beta=-$ $0.142, P<0.001)$. Furthermore, this association remained significant after additional adjustment of glycated haemoglobin A1c (HbA1c), triglyceride (TG), highdensity lipoprotein cholesterol (HDL-C), low-density lipoprotein cholesterol (LDL-C), ALT, AST, and GGT (standardized $\beta=-0.059, P=0.042$ ).

Table 1 Characteristic of the study subjects

\begin{tabular}{|c|c|c|c|}
\hline Variables & Total $(n=1206)$ & Non-FDR $(n=957)$ & $\operatorname{FDR}(n=249)$ \\
\hline Age (years) & $59.7(54.8-64.3)$ & $59.9(54.8-64.4)$ & $59.0(54.0-63.2)$ \\
\hline Men, n (\%) & $413(34.2)$ & $337(35.2)$ & $76(30.5)$ \\
\hline $\mathrm{BMI}\left(\mathrm{kg} / \mathrm{m}^{2}\right)$ & $24.0 \pm 3.2$ & $24.0 \pm 3.2$ & $24.2 \pm 3.3$ \\
\hline$W(\mathrm{~cm})$ & $82.0(77.0-89.0)$ & $82.0(77.0-88.0)$ & $83.0(77.0-89.8)$ \\
\hline $\mathrm{SBP}(\mathrm{mmHg})$ & $129.0(118.0-141.0)$ & $130.0(119.0-142.0)$ & $124.0(114.0-137.0)^{* *}$ \\
\hline $\mathrm{DBP}(\mathrm{mmHg})$ & $77.0(70.0-84.0)$ & $77.0(71.0-85.0)$ & $75.0(68.0-82.0)^{* *}$ \\
\hline FPG (mmol/L) & $5.7 \pm 0.5$ & $5.7 \pm 0.5$ & $5.7 \pm 0.5$ \\
\hline $2 \mathrm{hPG}(\mathrm{mmol} / \mathrm{L})$ & $7.0 \pm 1.8$ & $7.0 \pm 1.7$ & $7.2 \pm 1.8^{*}$ \\
\hline $\mathrm{HbA1c}(\%)$ & $5.6(5.4-5.9)$ & $5.6(5.4-5.8)$ & $5.7(5.5-5.9)^{* *}$ \\
\hline HOMA-IR & $2.2(1.5-3.2)$ & $2.2(1.5-3.1)$ & $2.2(1.5-3.3)$ \\
\hline TC (mmol/L) & $5.4(4.8-6.0)$ & $5.4(4.8-6.0)$ & $5.3(4.8-6.1)$ \\
\hline $\mathrm{TG}(\mathrm{mmol} / \mathrm{L})$ & $1.4(1.0-1.9)$ & $1.4(1.0-1.9)$ & $1.4(1.1-2.0)$ \\
\hline $\mathrm{HDL}-\mathrm{C}(\mathrm{mmol} / \mathrm{L})$ & $1.4(1.2-1.7)$ & $1.4(1.2-1.7)$ & $1.4(1.2-1.7)$ \\
\hline LDL-C (mmol/L) & $3.3(2.8-3.8)$ & $3.3(2.8-3.8)$ & $3.3(2.8-3.9)$ \\
\hline CRP (mg/L) & $0.9(0.4-1.6)$ & $0.9(0.4-1.6)$ & $0.9(0.5-1.7)$ \\
\hline $\mathrm{ALT}(\mathrm{U} / \mathrm{L})$ & $18.0(14.0-24.0)$ & $18.0(14.0-24.0)$ & $18.0(14.0-24.0)$ \\
\hline AST (U/L) & $20.0(18.0-24.0)$ & $20.0(18.0-24.0)$ & $20.0(17.5-24.0)$ \\
\hline GGT (U/L) & $22.0(17.0-31.0)$ & $22.0(17.0-32.0)$ & $22.0(16.0-30.0)$ \\
\hline LFC (\%) & $8.2(6.6-22.2)$ & $8.2(6.6-22.6)$ & $8.4(6.6-16.2)$ \\
\hline NAFLD, n (\%) & $479(39.7)$ & $379(39.6)$ & $100(40.2)$ \\
\hline Impaired glucose regulation, n (\%) & $513(42.5)$ & $392(41.0)$ & $121(48.6)^{*}$ \\
\hline
\end{tabular}

Continuous variables are expressed as means \pm standard deviation or medians with interquartile range. Categorical variables are expressed as numbers with percentages

FDR versus non-FDR, ${ }^{*} P<0.05,{ }^{* *} P<0.01$

FDR first degree relatives of patients with diabetes, $B M I$ body mass index, $W$ waist circumference, SBP systolic blood pressure, $D B P$ diastolic blood pressure, $F P G$ fasting plasma glucose, 2 PPG 2-h plasma glucose, HbA1c glycated hemoglobin A1c, HOMA-IR homeostasis model assessment-insulin resistance index, TC total cholesterol, $T G$ triglyceride, $H D L-C$ high-density lipoprotein cholesterol, $L D L-C$ low-density lipoprotein cholesterol, CRP C-reactive protein, $A L T$ alanine aminotransferase, AST aspartate aminotransferase, GGT gamma-glutamyl transpeptidase, NAFLD non-alcoholic fatty liver disease 


\section{Comparison of serum osteocalcin levels according to the presence of FDR and NAFLD}

As shown in Fig. 1, serum osteocalcin levels were significantly lower in FDR than in non-FDR $(19.8 \pm 5.7 \mathrm{ng} / \mathrm{mL}$ versus $20.7 \pm 6.8 \mathrm{ng} / \mathrm{mL}, P=0.028$ ). We further divided the subjects into four groups according to the presence of FDR and NAFLD: group 1, non-FDR + non-NAFLD; group 2, FDR + non-NAFLD; group 3, non-FDR + NAFLD; and group 4, FDR + NAFLD. Figure 2 shows that serum osteocalcin levels were reduced in subjects with NAFLD whether accompanied by FDR or not $(P=$ 0.012; $P=0.003)$. In addition, FDR had a lower concentration of serum osteocalcin than non-FDR among subjects with NAFLD $(P=0.011)$. Serum osteocalcin levels showed a decreasing trend from group 1 to group 4 ( $P$ for trend $<0.001$ ).

\section{Relationship between serum osteocalcin levels and FDR}

A multivariate linear regression analysis was performed to explore the independent relationship between serum osteocalcin levels and FDR. As shown in Table 2, it was found that the presence of FDR was negatively associated with serum osteocalcin levels after adjusting for age, gender, and menopausal status (in women) in model 1 (standardized $\beta=-0.067, P=0.010$ ). In model 2 , after further adjusting for BMI, W, systolic blood pressure, diastolic blood pressure, HbA1c, HOMA-IR, TG, HDL$\mathrm{C}$, LDL-C, and C-reactive protein (CRP), the association remained (standardized $\beta=-0.055, P=0.031$ ). In model 3 , the analysis confirmed that after further adjustment of LFC and previous factors, the presence of FDR was an independent determinant of serum osteocalcin levels (standardized $\beta=-0.057, P=0.028$ ).

\section{Discussion}

In the present study, not only in subjects with NAFLD but also in FDR, the levels of serum osteocalcin went down significantly. Furthermore, FDR exhibited lower serum osteocalcin levels than non-FDR among those with NAFLD. An inverse correlation between serum osteocalcin levels and the presence of FDR remained significant after adjusting for relevant factors and LFC.

In recent studies, bone is recognised as a biologically active organ. Osteocalcin is one of the proteins that play a role in regulating insulin secretion and increasing insulin sensitivity in peripheral tissues. We have found that in both men and women, subjects with serum osteocalcin levels below the median had higher HOMA-IR values compared with those with levels above the median [19]. Moreover, previous studies observed that serum osteocalcin levels were closely related to metabolic diseases due to insulin resistance [17, 20, 21]. The concentrations of serum osteocalcin were significantly lower in NAFLD patients $[11,12]$. Lower serum osteocalcin levels were associated with the presence of NAFLD, even in obese people [22]. However, the prediction of LFC in the above studies was based on a model calculated by routine laboratory indicators. Although liver biopsy remains the gold standard for the diagnosis of NAFLD, its value for community-based studies is limited because of its invasive nature. Recent studies using imaging examination not only noninvasively but also

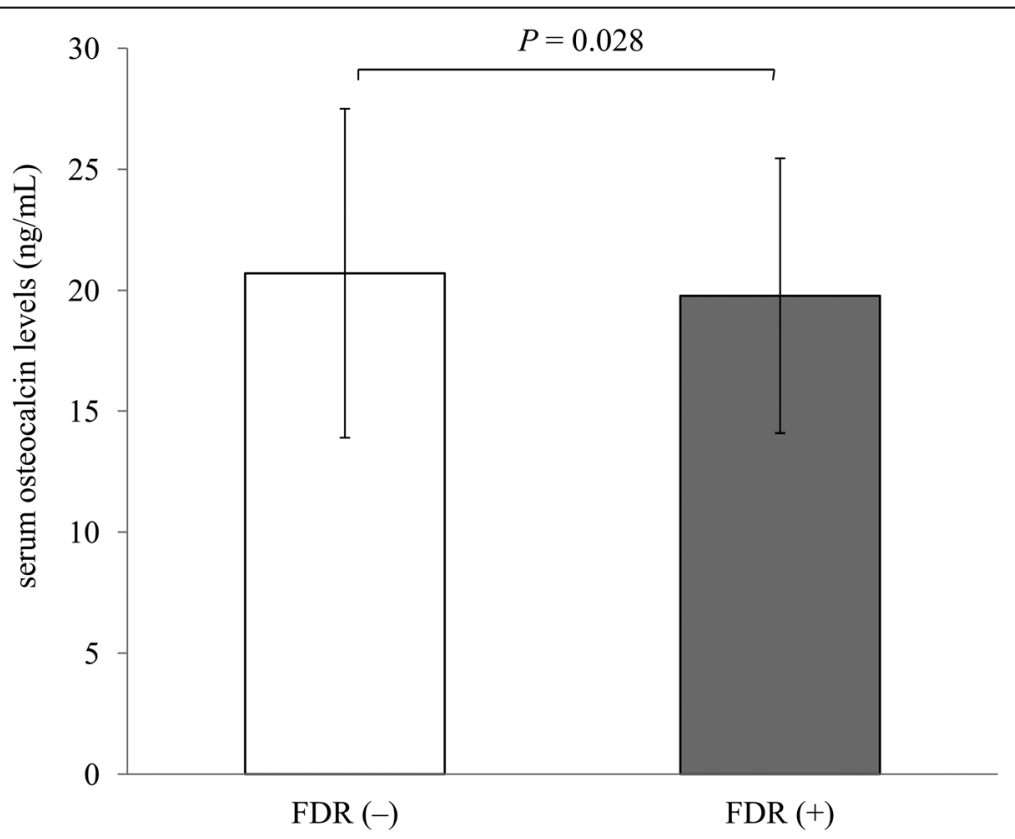

Fig. 1 Association of serum osteocalcin levels with FDR. The variables and error bars are shown as mean with standard deviation 


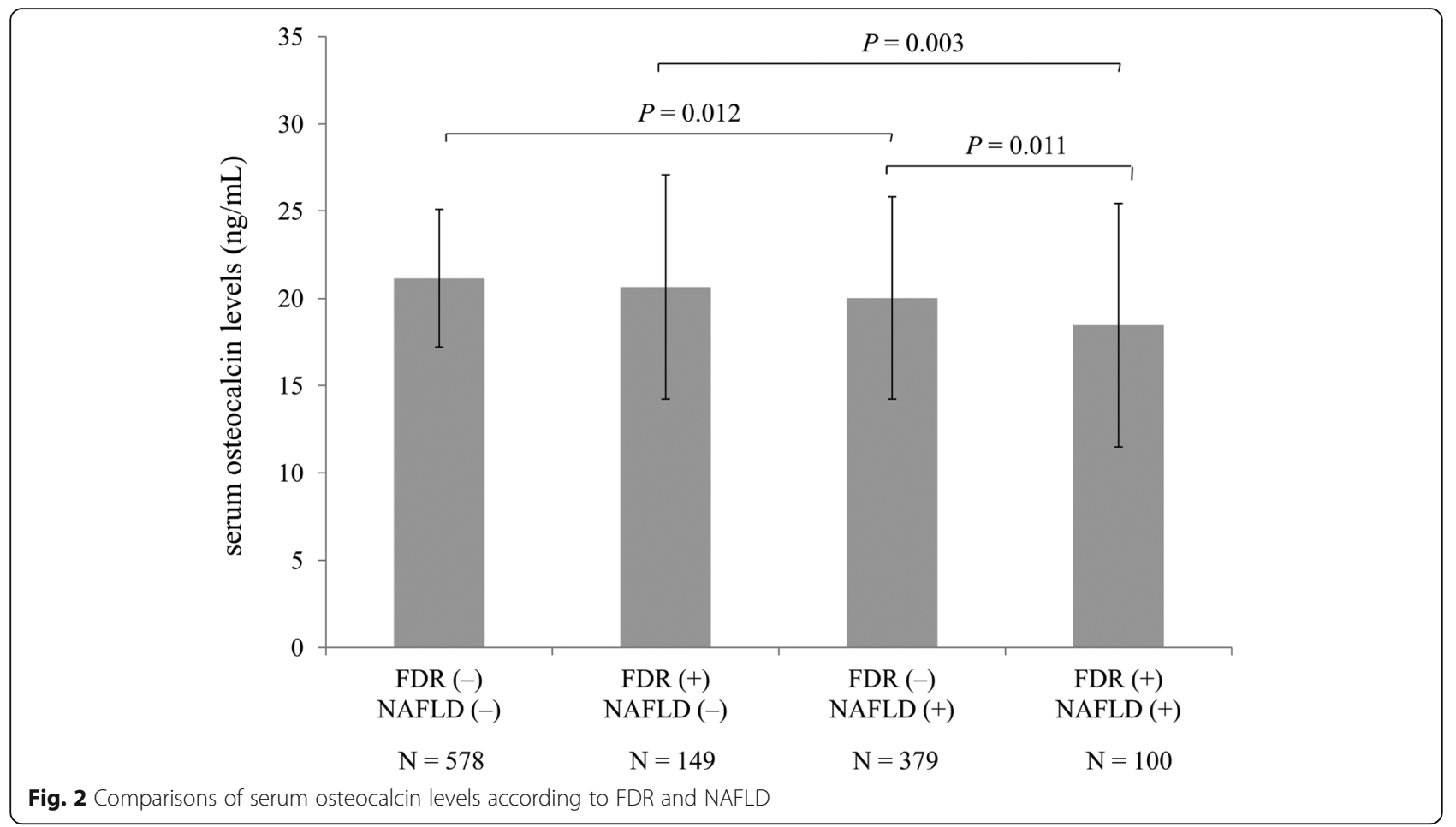

accurately estimated LFC; in particular, because of its convenience, ultrasound technology is being developed and applied to research conducted in populations with a large sample. LFC has been involved in some clinical studies related to metabolic abnormalities, atherosclerosis, and cytokines [14]. The present study excluded subjects with diabetes or subjects undergoing lipidlowering treatments to rule out the influence on serum osteocalcin levels or LFC. We observed a significant negative correlation between serum osteocalcin levels and LFC. Furthermore, using the quantitative cut-off point, it was found that serum osteocalcin levels decreased in subjects with NAFLD, which was consistent

Table 2 Multiple-adjusted associations of FDR and serum osteocalcin levels

\begin{tabular}{llll}
\hline FDR & \multicolumn{3}{l}{ Serum osteocalcin levels } \\
\cline { 2 - 4 } & standardized $\beta$ & $\mathrm{t}$ & $\mathrm{P}$ \\
\hline Model 1 & -0.067 & -2.576 & 0.010 \\
Model 2 & -0.055 & -2.154 & 0.031 \\
Model 3 & -0.057 & -2.205 & 0.028 \\
\hline
\end{tabular}

Model 1: adjusted for age, gender and menopause status (in women) Model 2: adjusted for age, gender, menopause status (in women), BMI, W, SBP, DBP, HbA1C, HOMA-IR, TG, HDL-C, LDL-C, and CRP

Model 3: adjusted for age, gender, menopause status (in women), BMI, W, SBP, DBP, HbA1C, HOMA-IR, TG, HDL-C, LDL-C, CRP and LFC

$F D R$ first degree relatives of patients with diabetes, $B M I$ body mass index, $W$ waist circumference, SBP systolic blood pressure, $D B P$ diastolic blood pressure, $H b A 1 c$ glycated hemoglobin A1c, HOMA-IR homeostasis model assessmentinsulin resistance index, $T G$ triglyceride, $H D L-C$ high-density lipoprotein cholesterol, $L D L-C$ low-density lipoprotein cholesterol, CRP C-reactive protein, LFC liver fat content with the previous studies. Recent studies have demonstrated a protective effect of osteocalcin against NAFLD, and our previous studies suggested that osteocalcin could improve NAFLD by alleviating oxidative stress and probably by modulating insulin signaling pathway or hepatic lipid metabolism, although the specific underlying mechanisms need to be confirmed with further studies.

In addition, serum osteocalcin levels were also lower in FDR than non-FDR, and the association still existed among subjects with NAFLD. The negative correlation of FDR with serum osteocalcin levels was not influenced by metabolic factors such as BMI, blood glucose, lipids, and LFC. The demonstration of an endocrine feedback loop implied that osteocalcin acted as a hormone improving insulin sensitivity and stimulating insulin secretion; however, its secretion and bioactivity were regulated by insulin signalling. Therefore, the relationship between FDR and serum osteocalcin levels was possibly mediated by insulin resistance.

Ishikawa et al. revealed that FDR tended to be at high risk of developing insulin resistance, and hyperinsulinemia was present even in the absence of obesity [23]. A European multicentre study provided conclusive evidence of the presence of insulin resistance in nondiabetic FDR, even after correction for various covariates [24]. FDR had a defective insulin activation compared with matched controls, and what was worse, insulin sensitivity began to fall from childhood [25]. A study from South Korea also observed a higher prevalence of 
HOMA-IR in FDR even in the presence of a normal glycaemic profile [26]. Other clinical studies demonstrated that in FDR, insulin resistance, beta-cell dysfunction, and adipocyte dysfunction exhibited altered trends prior to the development of impaired glucose tolerance or diabetes [27].

The potential mechanisms related to the association between FDR and serum osteocalicn levels remain unknown. The presence of insulin resistance in FDR may be an explanation. Wei et al. reported that one molecular mechanism that reduced bone resorption due to insulin resistance leads to decreased insulin signalling in osteoblasts, which caused a reduction in the circulating levels of the bone-derived hormone osteocalcin [28]. The active form of osteocalcin in mice fed a high-fat diet was lower than in the control [28]. Moreover, insulin receptor signalling in osteoblasts controlled osteoblast development and osteocalcin expression. Mice that lacked the insulin receptor had lower circulating osteocalcin levels [29]. Other studies have confirmed the negative effect of insulin resistance on the osteocalcin gene in the osteoblast in vitro [30].

There were several limitations in this study. First, we could not fully elucidate the causal relationship between serum osteocalcin levels and FDR. The role of family history of diabetes in serum osteocalcin levels remains to be explored in further studies. Sceond, the family history of diabetes was self-reported, though accuracy of self-reported family history is highest for first-degree relatives. Third, due to lacking an automated assay to determine under-carboxylated osteocalcin, we only measured its total form, which were proved to be correlated with energy metabolism. In addition, vitamin $\mathrm{K}$ was not measured in this study, though circulating osteocalcin levels may be influenced by Vitamin K.

\section{Conclusion}

We found the lower levels of serum osteocalcin in FDR than in non-FDR, which persisted in subjects with NAFLD. The relationship between FDR and serum osteocalcin levels was independent of metabolic factors such as blood glucose, lipids, and LFC.

\footnotetext{
Abbreviations

2hPG: 2-h plasma glucose; ALT: alanine aminotransferase; AST: aspartate aminotransferase; BMI: body mass index; CRP: C-reactive protein; FDR: first degree relatives of patients with diabetes; FINS: fasting serum insulin; FPG: fasting plasma glucose; GGT: gamma-glutamyl transpeptidase; HbA1c: glycated haemoglobin A1c; HDL-C: high-density lipoprotein cholesterol; HOMA-IR: homeostasis model assessment-insulin resistance index; LDL-C: low-density lipoprotein cholesterol; LFC: liver fat content; NAFLD: non-alcoholic fatty liver disease; TG: triglyceride; W: waist circumference
}

\section{Acknowledgements}

None.

\section{Authors' contributions}

XJM and YQB conceived and designed the experiments. YTX, YS, CCG and YFW performed the experiments. YTX and YS wrote the paper. All authors read and approved the final manuscript.

\section{Funding}

This work was supported by the Project of National Natural Science Foundation of China (31571212) and the funders did not play any role in the design of the study and collection, analysis and interpretation of data and in writing the manuscript.

\section{Availability of data and materials \\ The dataset used and analysed during the current study are available from the corresponding author on reasonable request.}

\section{Ethics approval and consent to participate}

This study was conducted according to the World Medical Association Declaration of Helsinki and approved by the Ethics Committee of Shanghai Jiao Tong University Affiliated Sixth People's Hospital. All subjects provided written informed consent before the interview.

\section{Consent for publication}

Not applicable.

\section{Competing interests}

The authors declare that they have no competing interests.

\section{Received: 12 February 2019 Accepted: 11 November 2019}

\section{2.}

\section{References}

1. Beck-Nielsen H, Vaag A, Poulsen P, Gaster M. Metabolic and genetic influence on glucose metabolism in type 2 diabetic subjects--experiences from relatives and twin studies. Best Pract Res Clin Endocrinol Metab. 2003; 17:445-67.

2. Tam CH, Wang Y, Luan J, Lee HM, Luk AO, Tutino GE, et al. Maternal history of diabetes is associated with increased cardiometabolic risk in Chinese. Nutr Diabetes. 2014;4:e112.

3. Jenkins AB, Batterham M, Samocha-Bonet D, Tonks K, Greenfield JR, Campbell LV. Segregation of a latent high adiposity phenotype in families with a history of type 2 diabetes mellitus implicates rare obesitysusceptibility genetic variants with large effects in diabetes-related obesity. PLoS One. 2013;8:e70435.

4. Kubaszek A, Pihlajamäki J, Komarovski V, Lindi V, Lindström J, Eriksson J, et al. Finnish Diabetes Prevention Study. Promoter polymorphisms of the TNF-alpha (G-308A) and IL-6 (C-174G) genes predict the conversion from impaired glucose tolerance to type 2 diabetes: the Finnish Diabetes Prevention Study. Diabetes. 2003:52:1872-6.

5. Hu X, Pan X, Ma X, Luo Y, Xu Y, Xiong Q, et al. Contribution of a first-degree family history of diabetes to increased serum adipocyte fatty acid binding protein levels independent of body fat content and distribution. Int J Obes. 2016:40:1649-54.

6. Zhou M, Ma X, Li H, Pan X, Tang J, Gao Y, et al. Serum osteocalcin concentrations in relation to glucose and lipid metabolism in Chinese individuals. Eur J Endocrinol. 2009;161(5):723-9.

7. Wang J, Yan DD, Hou XH, Bao YQ, Hu C, Zhang ZL, et al. Association of bone turnover markers with glucose metabolism in Chinese population. Acta Pharmacol Sin. 2017;38(12):1611-7.

8. Dou J, Li H, Ma X, Zhang M, Fang Q, Nie M, et al. Osteocalcin attenuates high fat diet-induced impairment of endothelium-dependent relaxation through Akt/eNOS-dependent pathway. Cardiovasc Diabetol. 2014;13:74.

9. Lee NK, Sowa H, Hinoi E, Ferron M, Ahn JD, Confavreux C, et al. Endocrine regulation of energy metabolism by the skeleton. Cell. 2007;130(3):456-69.

10. Bullen JW Jr, Bluher S, Kelesidis T, Mantzoros CS. Regulation of adiponectin and its receptors in response to development of diet-induced obesity in mice. Am J Physiol Endocrinol Metab. 2007;292(4):E1079-86.

11. Dou J, Ma X, Fang Q, Hao Y, Yang R, Wang F, et al. Relationship between serum osteocalcin levels and non-alcoholic fatty liver disease in Chinese men. Clin Exp Pharmacol Physiol. 2013;40:282-8.

12. Luo YQ, Ma XJ, Hao YP, Pan XP, Xu YT, Xiong Q, et al. Inverse relationship between serum osteocalcin levels and nonalcoholic fatty liver disease in 
postmenopausal Chinese women with normal blood glucose levels. Acta Pharmacol Sin. 2015;36:1497-502.

13. Xia MF, Yan HM, He WY, Li XM, Li CL, Yao XZ, et al. Standardized ultrasound hepatic/renal ratio and hepatic attenuation rate to quantify liver fat content: an improvement method. Obesity (Silver Spring). 2012;20:444-52.

14. Xia MF, Bian H, Yan HM, Lin HD, Chang XX, Li XM, et al. Assessment of liver fat content using quantitative ultrasonography to evaluate risks for metabolic diseases. Obesity (Silver Spring). 2015;23:1929-37.

15. Xu Y, Ma X, Pan X, He X, Xiao Y, Bao Y. Correlations between serum concentration of three bone-derived factors and obesity and visceral fat accumulation in a cohort of middle aged men and women. Cardiovasc Diabetol. 2018;17:143.

16. Cederberg H, Stančáková A, Kuusisto J, Laakso M, Smith U. Family history of type 2 diabetes increases the risk of both obesity and its complications: is type 2 diabetes a disease of inappropriate lipid storage? J Intern Med. 2015; 277:540-51.

17. Bao Y, Ma X, Yang R, Wang F, Hao Y, Dou J, et al. Inverse relationship between serum osteocalcin levels and visceral fat area in Chinese men. J Clin Endocrinol Metab. 2013;98:345-51.

18. Department of Noncommunicable Disease Surveillance. Report of a WHO consultation: definition, diagnosis and classification of diabetes mellitus and its complication. Part 1: Diagnosis and classification of diabetes mellitus. Geneva: World Health Organization; 1999

19. Zhang X, Shen Y, Xu Y, Xiong Q, Lu Z, Ma X, et al. Association of serum osteocalcin levels with major adverse cardiovascular events: a 4.4-year retrospective cohort study. Clin Exp Pharmacol Physiol. 2018:45:3-9.

20. Luo Y, Ma X, Hao Y, Xu Y, Xiong Q, Tang J, et al. Association between serum osteocalcin level and visceral obesity in Chinese postmenopausal women. Clin Endocrinol. 2015;83:429-34

21. Du J, Zhang M, Lu J, Zhang X, Xiong Q, Xu Y, et al. Osteocalcin improves nonalcoholic fatty liver disease in mice through activation of Nrf2 and inhibition of JNK. Endocrine. 2016;53:701-9.

22. Liu JJ, Chen YY, Mo ZN, Tian GX, Tan AH, Gao Y, et al. Relationship between serum osteocalcin levels and non-alcoholic fatty liver disease in adult males, South China. Int J Mol Sci. 2013;14:19782-91.

23. Ishikawa M, Pruneda ML, Adams-Huet B, Raskin P. Obesity-independent hyperinsulinemia in nondiabetic first-degree relatives of individuals with type 2 diabetes. Diabetes. 1998;47:788-92.

24. Vaag A, Lehtovirta M, Thye-Rönn P. Groop L, European Group of Insulin Resistance. Metabolic impact of a family history of type 2 diabetes. Results from a European multicentre study (EGIR). Diabet Med. 2001;18:533-40.

25. Arslanian SA, Bacha F, Saad R, Gungor N. Family history of type 2 diabetes is associated with decreased insulin sensitivity and an impaired balance between insulin sensitivity and insulin secretion in white youth. Diabetes Care. 2005;28:115-9.

26. Moon JH, Roh E, Oh TJ, Kim KM, Moon JH, Lim S, et al Increased risk of metabolic disorders in healthy young adults with family history of diabetes: from the Korea National Health and nutrition survey. Diabetol Metab Syndr. 2017:9:16.

27. Stadler M, Pacini G, Petrie J, Luger A, Anderwald C, RISC Investigators. Beta cell (dys)function in non-diabetic offspring of diabetic patients. Diabetologia. 2009;52:2435-44.

28. Wei J, Ferron M, Clarke CJ, Hannun YA, Jiang H, Blaner WS, et al. Bonespecific insulin resistance disrupts whole-body glucose homeostasis via decreased osteocalcin activation. J Clin Invest. 2014:124:1-13.

29. Fulzele K, Riddle RC, DiGirolamo DJ, Cao X, Wan C, Chen D, et al. Insulin receptor signaling in osteoblasts regulates postnatal bone acquisition and body composition. Cell. 2010;142:309-19.

30. Bilotta FL, Arcidiacono B, Messineo S, Greco M, Chiefari E, Britti D, et al. Insulin and osteocalcin: further evidence for a mutual cross-talk. Endocrine. 2018:59:622-32.

\section{Publisher's Note}

Springer Nature remains neutral with regard to jurisdictional claims in published maps and institutional affiliations.

\section{Ready to submit your research? Choose BMC and benefit from:}

- fast, convenient online submission

- thorough peer review by experienced researchers in your field

- rapid publication on acceptance

- support for research data, including large and complex data types

- gold Open Access which fosters wider collaboration and increased citations

- maximum visibility for your research: over $100 \mathrm{M}$ website views per year

At BMC, research is always in progress.

Learn more biomedcentral.com/submissions 